\title{
Re-exploration Early after Cardiac Surgery in Adults: The Importance of Bleeding-Related Complications
}

\author{
Ioannis G. Bougioukas, Martin G. Friedrich, Bernhard C. Danner, Friedrich A. Schoendube, \\ Theodor Tirilomis \\ Department of Thoracic, Cardiac, and Vascular Surgery, University of Göttingen, Göttingen, Germany
}

\section{ABSTRACT}

Background: Re-explorations soon after cardiac surgery are mostly related to bleeding or unclear hemodynamic situations possibly related to heart compression resulting from pericardial hematoma. This condition has a significant impact on mortality, morbidity, and costs. The aim of this study was to analyze indications and outcomes of re-exploration for bleeding or pericardial tamponade early after cardiac surgery in adults.

Methods: The clinical data of 4790 consecutive adult patients who underwent cardiac surgery in our institution from January 2011 to May 2016 were retrospectively analyzed.

Results: We identified 331 re-explorations performed in 231 patients. Sixty-seven of these patients had $>1$ reexploration. In most cases $(88 \%)$, repeat sternotomy was performed. Most procedures (57\%) were performed within the first 48 hours. In two-thirds of re-explorations, active bleeding or pericardial hematoma was verified. In the remaining cases, neither bleeding nor significant pericardial hematoma leading to tamponade was found. Among the cases with active bleeding causes, the most bleeding sites were found to be at the coronary anastomosis and the epicardial exposure harvesting site, as well as from the side branches of bypass grafts and intercostal arteries.

Conclusions: The incidence of re-exploration after cardiac surgery in adults was low (4.8\%). In about two-thirds of the cases, active bleeding or significant pericardial hematoma was found. The most common bleeding causes were the easiest to treat.

\section{INTRODUCTION}

Re-explorations after cardiac surgery are rare but wellknown situations for the cardiac surgeon. Among the most common causes for re-exploration are acute bleeding and pericardial tamponade, as well as specific situations for coronary bypass revision or central placement of mechanical circulatory support.

Received February 2, 2020; accepted February 6, 2020.

Correspondence: Theodor Tirilomis, MD, PhD, MHBA, Dept. of Thoracic, Cardiac, and Vascular Surgery, University of Göttingen, Robert-Koch-Str. 40, 37075 Göttingen, Germany; 495513966004; fax: 495513933029 (e-mail: theodor.tirilomis@med.uni-goettingen.de).
Although coagulation diagnostics and management have improved over the years, perioperative bleeding is still a significant problem in cardiac surgery [Ghavidel 2015; Colson 2016; Perek 2016]. Bleeding tendency is probably inevitable these days, as recommendations of the latest guidelines, for example, advise operating while patients are on aspirin, and the growing use of novel oral anticoagulants that do not demand regular screening obfuscates the issue [Windecker 2014; Heidbuchel 2015]. In addition, other factors, such as patient-related or procedural factors, play an important role in the development of bleeding-related complications [Kristensen 2012].

Active bleeding with unexpected increased blood loss through the chest tubes is visible and the indication for reexploration quite easy, but sometimes bleeding is not so pronounced that re-exploration is required immediately. However, the role of such less-pronounced bleeding is not clear in the context of impaired heart function and probably results from pericardial hematoma.

Postoperative bleeding is associated with increased mortality [Ranucci 2013]. In addition, hospital stays are prolonged for patients undergoing re-exploration for bleeding [Hall 2001], and reopening a sternotomy wound early after initial surgery increases the risk for wound infection [Meszaros 2016].

The aim of this study was to gather our experience with reexplorations early after cardiac surgery, identify the bleeding cause, verify the indication for the re-exploration, and analyze the outcome.

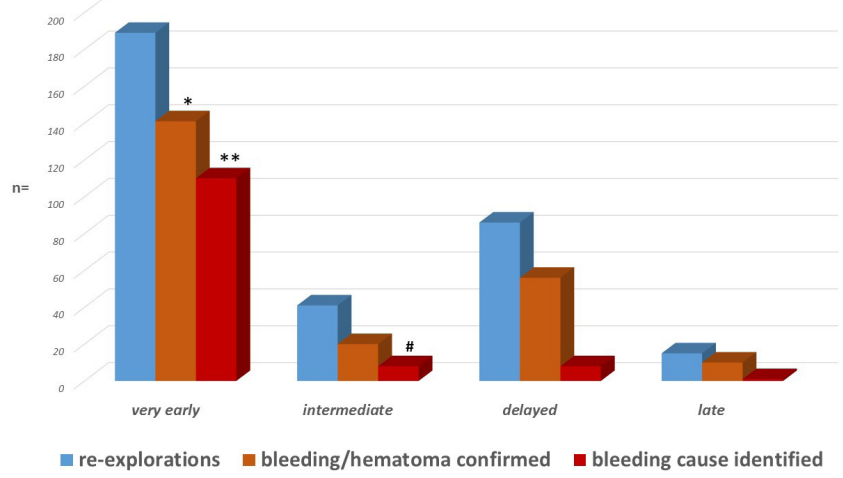

Re-explorations by time period. The confirmation rate and the causeof-bleeding identification rate were significant higher in the very early period after surgery. ${ }^{*} P<.001$ versus intermediate; $* * P<.001$ versus all other groups; $\# P<.05$ versus delayed. 
Cause of bleeding identified during re-exploration after cardiac surgery $(n=127)$

\begin{tabular}{|c|c|}
\hline Cause & $\mathrm{n}$ \\
\hline \multicolumn{2}{|c|}{ Anastomosis-related and graft-related bleeding } \\
\hline Distal graft anastomosis & 16 \\
\hline Proximal graft anastomosis & 17 \\
\hline Epi-/myocardial harvesting site & 15 \\
\hline Venous graft side branch & 11 \\
\hline Internal mammary artery side branch & 11 \\
\hline Internal mammary artery bed & 6 \\
\hline Vein graft laceration & 1 \\
\hline \multicolumn{2}{|l|}{ Cannulation-related lesions } \\
\hline Arterial cannulation site & 5 \\
\hline Venous cannulation site & 5 \\
\hline ECMO/ECLS arterial cannulation site & 1 \\
\hline ECMO/ECLS venous cannulation site & 2 \\
\hline Aortic needle vent site & 2 \\
\hline Left ventricular venting site & 2 \\
\hline \multicolumn{2}{|l|}{ Other cardiovascular and vascular lesions } \\
\hline Jugular vein laceration & 4 \\
\hline Left innominate vein laceration & 2 \\
\hline Left atrial appendix occlusion site & 2 \\
\hline Left atrial catheter dislocation & 2 \\
\hline Left atrial roof laceration & 1 \\
\hline Left ventricular wall laceration & 1 \\
\hline Aortotomy & 1 \\
\hline \multicolumn{2}{|l|}{ Other lesions } \\
\hline Intercostal artery & 11 \\
\hline Pericardial edge & 6 \\
\hline Sternal periosteal vessels & 3 \\
\hline Pacemaker wires & 1 \\
\hline Lung injury & 1 \\
\hline
\end{tabular}

\section{METHODS}

The data of all consecutive operations performed from January 2011 to May 2016 were retrospectively obtained from the surgical database. Pediatric and congenital procedures were excluded from the study. We included all major cardiac procedures: coronary artery bypass grafting (CABG), valve surgery (conventional or minimally invasive, including trans-apical catheter-based aortic valve implantation [TAVI]), surgery of the thoracic aorta, heart transplantation, and various other procedures (eg, extended septal myectomy for hypertrophic obstructive cardiomyopathy). Reoperations for wound healing disorders, sternal wound infection, or secondary closure of a sternotomy wound were excluded from the study. Re-explorations only for cannula removal of extracorporeal membrane oxygenation/life-support systems (ECMO/ ECLS) were also excluded, whereas procedures for complications were included.

The variables used for the database search were bleeding, mediastinal bleeding, mediastinal hematoma, reoperation for bleeding, pericardial tamponade, hemothorax, and hematoma evacuation. The approach variables were repeat sternotomy, re-sternotomy, thoracotomy, video-assisted thoracoscopic surgery (VATS), and subxiphoid pericardial drainage insertion.

After gathering and studying all data, we first categorized the types of primary cardiac procedures and their urgency. Then we aimed to verify the indication for re-exploration. If the indication was confirmed, we further clarified whether there was an identified cause of bleeding or a diffuse bleeding tendency. Active bleeding or other was defined by the surgeon performing the re-exploration.

The timing of postoperative re-exploration was categorized according to the date of performance: very early, within the day of surgery and the first postoperative day; intermediate, from postoperative day 2 to 4; and delayed, from postoperative day 5 to 30 . For some rare cases with possible bleeding-related problems after the early time of surgery, we also defined a late period: postoperative day 31 and after. Additionally, we correlated timing of re-exploration and identification of bleeding cause. Finally, the outcomes of patients who underwent re-exploration were analyzed.

Quantitative data were described by mean \pm standard deviation. Statistical analysis was carried out using SPSS software (17th edition; SPSS, Inc., Chicago, IL). $P$ values <.05 were considered significant.

\section{RESULTS}

In the study period, 4790 major cardiac procedures were performed in adults; 231 patients $(4.8 \%)$ underwent 331 reexplorations for postoperative suspicion of bleeding-related complications. Sixty-seven patients of those $231(29 \%)$ had $>1$ re-exploration (up to 4).

The initial cardiac procedures of the 231 patients who underwent re-exploration after surgery were CABG in ( $\mathrm{n}=$ $79 ; 34.2 \%)$; valve surgery $(\mathrm{n}=35 ; 15 \%)$ including minimal invasive surgery and trans-apical TAVI $(\mathrm{n}=10)$; combined procedures $(\mathrm{n}=95 ; 41.1 \%)$; and other procedures $(\mathrm{n}=22$; $9.5 \%)$. Sixteen patients $(7 \%)$ had a redo procedure requiring re-sternotomy. After the primary procedure, the sternotomy was left open in 32 patients (14\%), and 25 patients $(10.8 \%)$ were postoperatively on mechanical circulatory support (ECLS/ECMO). The primary surgery was elective in 144 patients $(62.3 \%)$, urgent in $56(24.2 \%)$, and emergency in 31 (13.5\%).

The majority of re-explorations were performed via repeat sternotomy ( $\mathrm{n}=291 ; 88 \%)$. A subxiphoid approach with pericardial drainage was performed in 24 cases. The remaining 16 cases underwent a lateral thoracotomy, including videoassisted thoracoscopic procedure $(n=6)$. 
More than half the re-explorations were performed in the very early postoperative period (189 reoperations, 57\%) and $41(12.5 \%)$ in the intermediate period (Figure). In 9 of the 15 cases in the late phase, only a subxiphoid pericardial drainage was placed.

Active bleeding or pericardial hematoma could be confirmed in 229 procedures (69\%). Active bleeding was identified in 127 of 229 cases $(55.5 \%)$, whereas in the remaining cases, bleeding had ceased at the time of reoperation and pericardial hematoma was evacuated. In one-third of cases $(31 \%)$ there was neither active bleeding nor hematoma. The highest confirmation rate of bleeding-related complication was in the very early phase after the primary surgery (Figure). Compared with the intermediate group, this finding was statistically significant $(75 \%$ in very early versus $49 \%$ in intermediate; $P<.001)$. The identification rate of the bleeding cause in the very early group, compared with all other groups, was also significantly higher $(P<.001)$ (Figure).

The analysis of identified bleeding causes $(n=127)$ revealed anastomosis-related and graft-related sources of bleeding (Table). Of the patients who underwent $>1$ re-exploration, 15 of 67 were on ECMO/ECLS support.

Of the 231 patients with re-exploration, 120 (52\%) had a satisfactory outcome without further complications in the postoperative course. Forty-four patients $(19 \%)$ had a complicated postoperative period with prolonged intensive care unit stay. Finally, 67 patients (29\%) died. The outcome of all patients on ECMO/ECLS who underwent $>1$ re-exploration was fatal.

\section{DISCUSSION}

Re-explorations for bleeding after cardiac surgery are common, with reported rates up to $16 \%$ [Haneya 2015]. In the present retrospective single-center analysis of 4790 consecutive adult patients who underwent cardiac surgery, we found an incidence of $4.8 \%$. All primary procedures-elective, urgent, or emergency-were included.

Usually, in uneventful cases after heart surgery without significant blood loss through the chest, tubes are removed on the second postoperative day. Thereafter, if indicated, antiplatelet treatment with aspirin is started, eg, in CABG patients.

Active bleeding is visible in surgical drainage. A defined amount of blood loss may facilitate the indication for reexploration [Colson 2016; Haneya 2015]. Nevertheless, the bleeding cause is not always localized during re-exploration. In many cases, the bleeding is diffuse, mostly from preoperative intake of aspirin or other antiplatelet drugs. Diffuse ("coagulopathic") bleeding has a worse acute outcome than surgical bleeding [Hall 2001]. More than one-third of the patients who underwent re-exploration in our study underwent the primary surgery in the urgent or emergency setting, while they were taking their anticoagulant treatment. Additionally, in elective cases, we did not stop aspirin administration before surgery. In cases of recent percutaneous interventions, patients were usually on dual antiplatelet therapy, according to the suggestions of interventional cardiologists.
More difficult is the situation in complex clinical cases with previous increased or reduced bleeding, as well as deterioration of pump function. Sometimes, echocardiography may help identify pericardial hematoma leading to tamponade. Because re-exploration delay increases risk of adverse outcome, in unclear situations we mostly decided in favor of re-exploration [Choong 2007; Karthik 2004]. Mortality was reported to be increased, up to 9 times, for patients with cardiac tamponade over patients with bleeding [Haneya 2015]. In this context, we could not identify active bleeding or significant pericardial hematoma in nearly one-third of the procedures. In present study, if the bleeding cause was identified, it was in most cases graft- and anastomosis-related. Bleeding related to cannulation sites was very rare, probably because of increased attention of surgeons during the primary surgery, knowing the possibly fatal consequences of bleeding from the aorta or the great vessels. Most of the bleeding causes were able to be managed relatively easily.

Of course, re-explorations were performed mostly in the very early and intermediate period postoperatively, and mainly through re-sternotomy. In general, the data analysis showed that the longer since the primary operation, the less clear is the cause of complication leading to re-exploration. Quite different was the situation in the so-called late period (after postoperative day 31), with the majority of cases treated via subxiphoid exploration and drainage. Although subxiphoid access does not allow complete inspection of the intrapericardial area, it is very helpful to identify the amount and quality of pericardial hematoma or effusion and may avoid reopening of the sternal wound $>1$ month after primary surgery.

In conclusion, most bleeding complications in the early postoperative phase are related to coronary bypass graft and sternal closure, and most of the bleeding causes were managed relatively easily. In nearly one-third of the reexplorations, active bleeding or significant pericardial hematoma was not found.

\section{ACKNOWLEDGMENT}

We acknowledge support by the German Research Foundation and the Open Access Publication Funds of the Göttingen University.

\section{REFERENCES}

Choong CK, Gerrard C, Goldsmith KA, Dunningham H, Vuylsteke A. Delayed re-exploration for bleeding after coronary artery bypass surgery results in adverse outcomes. Eur J Cardiothorac Surg 2007;31:834-838.

Colson PH, Gaudard P, Fellahi JL, et al. Active bleeding after cardiac surgery: A prospective observational multicentre study. PLoS One 2016;11:e0162396.

Ghavidel AA, Toutounchi Z, Shahandashti FJ, Mirmesdagh Y. Rotational thromboelastometry in prediction of bleeding after cardiac surgery. Asian Cardiovasc Thorac Ann 2015;23:525-529.

Hall TS, Brevetti GR, Skoultchi AJ, et al. Re-exploration for hemorrhage following open heart surgery differentiation on the causes of 
bleeding and the impact on patient outcomes. Ann Thorac Cardiovasc Surg 2001;7:352-357.

Haneya A, Diez C, Kolat P, et al. Re-exploration for bleeding or tamponade after cardiac surgery: Impact of timing and indication on outcome. Thorac Cardiovasc Surg 2015;63:51-57.

Heidbuchel H, Berti D, Campos M, et al. Implementation of non-vitamin $\mathrm{K}$ antagonist oral anticoagulants in daily practice: The need for comprehensive education for professionals and patients. Thromb J 2015;13:22.

Karthik S, Grayson AD, McCarron EE, Pullan DM, Desmond MJ. Re-exploration for bleeding after coronary artery bypass surgery: risk factors, outcomes, and the effect of time delay. Ann Thorac Surg 2004;78:527-534.

Kristensen KL, Rauer LJ, Mortensen PE, Kjeldsen BJ. Reoperation for bleeding in cardiac surgery. Interact Cardiovasc Thorac Surg 2012;14:709-713.
Meszaros K, Fuehrer U, Grogg S, et al. Risk factors for sternal wound infection after open heart operations vary according to type of operation. Ann Thorac Surg 2016;101:1418-1425.

Perek B, Stefaniak S, Komosa A, et al. Routine transfusion of platelet concentrates effectively reduces reoperation rate for bleeding and pericardial effusion after elective operations for ascending aortic aneurysm. Platelets 2016;2:1-7.

Ranucci M, Baryshnikova E, Castelvecchio S, Pelissero G. Major bleeding, transfusions, and anemia: The deadly triad of cardiac surgery. Ann Thorac Surg 2013;96:478-485.

Windecker S, Kolh P, Alfonso F, et al. 2014 ESC/EACTS Guidelines on myocardial revascularization: The Task Force on Myocardial Revascularization of the European Society of Cardiology (ESC) and the European Association for Cardio-Thoracic Surgery (EACTS) Developed with the special contribution of the European Association of Percutaneous Cardiovascular Interventions (EAPCI). Eur Heart J 2014;35:2541-2619. 